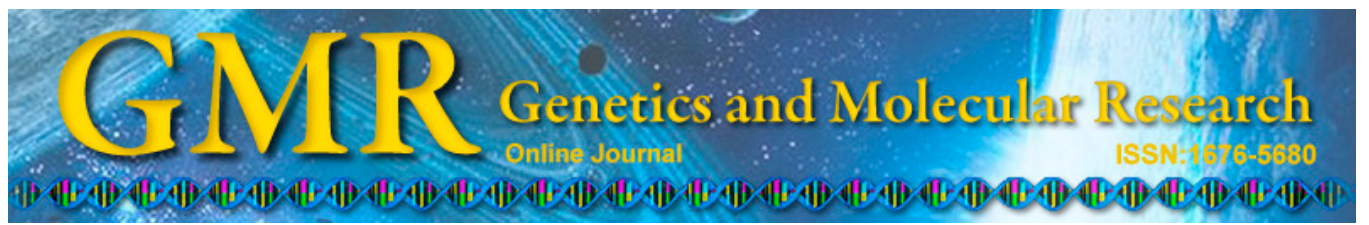

\title{
Clinical value of ultrasound-guided percutaneous biopsy of pulmonary lesions
}

\author{
R.J. Yang*, Y.L. Yang*, L.T. Ruan, L.J. Yuan, L.J. Chao, S. Chen and \\ Y.Y. Duan \\ Department of Ultrasound Diagnostics, Tangdu Hospital, \\ Fourth Military Medical University, Xi'an, Shaanxi Province, China \\ *These authors contributed equally to this study. \\ Corresponding author: Y.Y. Duan \\ E-mail: yunyouduan@126.com
}

Genet. Mol. Res. 14 (2): 3784-3790 (2015)

Received June 6, 2014

Accepted October 28, 2014

Published April 22, 2015

DOI http://dx.doi.org/10.4238/2015.April.22.7

\begin{abstract}
The purpose of this study was to evaluate the applicability of ultrasound-guided percutaneous biopsy for the diagnosis and differentiation of various pulmonary lesions in a Chinese population. A total of 338 pulmonary lesions were biopsied with 18-gauge cutting needles, guided by ultrasound, and sent for histopathological analysis. The ultrasonographic characteristics of these lesions, procedure complications, and histopathological diagnoses were analyzed. Sufficient specimen for histopathologic analysis was obtained in $95.64 \%$ (351/367), and mild complications occurred in $2.72 \%(10 / 367)$ of the patients. Accurate diagnosis was obtained in $94.03 \%$ (315/335) of the patients; 16 were lost to follow-up. Using the combination of shape and echogenicity to distinguish benign $v s$ malignant lesions, diagnostic sensitivity and specificity were 57.39 and $95.65 \%$, respectively. No significant difference was found between malignant and benign lesions in blood flow signals. Ultrasound-guided core biopsy is valuable for the diagnosis, management, and prognosis of unknown pulmonary lesions. Shape and echogenicity on ultrasonography correlate well with histopathology and provide useful information for distinguishing between
\end{abstract}


benign and malignant lesions. On the contrary, color Doppler is of little value for this purpose.

Key words: Pulmonary lesion; Ultrasound-guided biopsy; Color Doppler

\section{INTRODUCTION}

Pulmonary lesions can have various clinical and radiographic presentations and usually require biopsy for definitive diagnosis. Accurate etiologic diagnosis of pulmonary diseases is important for their subsequent management (Charboneau et al., 1990; Haramati, 1995; Shami and Waxman, 2005). However, surgical biopsy is complicated by the morbidity associated with the procedure. Ultrasonography has proved to be safe, accurate, portable, and inexpensive and does not require radiation exposure. Since the first report of successful ultrasound-guided percutaneous transthoracic needle biopsy of peripheral pulmonary masses in 1976 (Chandrasekhar et al., 1976), this technique has gradually been accepted as an alternative approach to fluoroscopic- and computed tomography (CT)-guided biopsy for the diagnosis of thoracic tumors (Seyfarth et al., 2007; Vilmann and Puri, 2007; Kennedy et al., 2008; Feller-Kopman et al., 2009; Chen et al., 2010; Beslic et al., 2012; Bugalho et al., 2013; Meng et al., 2013). Radiography and CT are the preferred modalities for diagnosing pulmonary lesions as benign $v s$ malignant. However, if pulmonary lesions could, instead, be diagnosed by ultrasonography, the necessity for biopsy procedures would be reduced, resulting in decreased emotional stress for patients. The purpose of this study was to evaluate ultrasonography and ultrasound-guided percutaneous biopsy for the diagnosis and differentiation of various peripheral pulmonary lesions in a Chinese population.

\section{MATERIAL AND METHODS}

\section{Study design, setting, and population}

Three hundred and sixty-seven patients (254 males and 113 females; aged from 7 months to 83 years, mean age $57 \pm 5$ years) with suspected pulmonary lesions on radiography, CT, and ultrasonography, between June 2007 and November 2011 at Tangdu Hospital, were included. Chest radiography and fiberoptic bronchoscopy were performed on each patient before the ultrasonographic study.

\section{Study protocol}

The Acuson Sequoia 512 ultrasound system with a 4V1 transducer and GE Logiq 5 ultrasound system with a 3.5C transducer were employed for ultrasound study. Characteristics of the lesions, including size, shape, echogenicity, and blood flow within the lesions were analyzed. Blood supplies within the lesions were categorized into 4 grades, from 0 to III, as follows: 0, no blood signal in the lesion; I, sparse dots of blood flow signal in the lesion; II, one blood vessel in the lesion; III, 2 or more blood vessels in the lesion. The lesions were grouped according to their ultrasonographic characteristics.

All biopsies were guided with real-time ultrasonography. Each transducer was equipped with a guiding channel that provided a preset puncture area displayed on the moni- 
tor screen. The biopsy route of the 4V1 transducer could be adjusted at a fixed angle of 18/32 degrees. The biopsy procedure was performed 1 to 4 times (mean 2.6) with an 18-gauge (1.2 $\mathrm{mm}$ ) Tru-Cut needle and a biopsy gun (Bard Biopsy Systems, USA) that had 2 alternative depths for drawing out the specimen, 15 and $22 \mathrm{~mm}$. Biopsy specimens were put directly into a formaldehyde solution (formalin) for histological analysis.

The confirmatory diagnosis of a pulmonary lesion was based on: 1) surgical resection or biopsy; 2) subsequent clinical course showing increase or decrease in size or disappearance of the lesion; and 3) clinical follow-up on the chest radiograph or CT scan.

All procedures were in accordance with the ethical standards of the World Medical Association (Declaration of Helsinki). The study was approved by the Ethics Committee and informed consents were obtained from all patients.

\section{Statistical analysis}

Data were processed with the SPSS 14.0 software, and chi-square criterion and rank sum test were used to compare differences among groups. $\mathrm{P}<0.05$ was considered to be statistically significant. The validity and reliability of ultrasonographic characteristics for diagnosis and differentiation of benign from malignant lesions were evaluated by comparison with final results.

\section{RESULTS}

The size of the lesions ranged between 1.2 and $9.5 \mathrm{~cm}$ (mean $4.15 \pm 1.97 \mathrm{~cm}$ ) measured by ultrasonography. Diagnoses of the pulmonary lesions are shown in Table 1.

Table 1. Histopathology and confirmative diagnosis in all the patients.

\begin{tabular}{lcc}
\hline & Histopathology & Final diagnosis \\
\hline Malignant disease & & \\
Squamous carcinoma & 86 & 94 \\
Adenocarcinoma & 75 & 83 \\
Adenosquamous carcinoma & 12 & 13 \\
Small-cell carcinoma & 10 & 18 \\
Alveolar epithelium carcinoma & 4 & 7 \\
Others & 20 & 20 \\
Benign disease & 35 & 32 \\
Tubercle & 94 & 73 \\
Chronic inflammation & 10 & 9 \\
Inflammatory pseudotumor & 5 & 2 \\
Others & 16 & 0 \\
Others & 0 & 16 \\
$\quad$ Inadequate specimen & & \\
Loss of follow-up & & \\
\hline
\end{tabular}

Sufficient specimen for histopathologic analysis was obtained from $95.64 \%(351 / 367)$ of the lesions. Samples that failed to provide sufficient histopathologic specimen were taken from areas of necrosis $(\mathrm{N}=3)$, tissue fragmentation $(\mathrm{N}=3)$, or collapsed lung $(\mathrm{N}=5)$. Additionally, some specimens were acquired outside of the target tissue $(\mathrm{N}=5)$. Accurate diagnosis was obtained in $94.03 \%(315 / 335)$ of the patients. Twenty malignant lesions were misdiagnosed as benign lesions.

Complications occurred in 10 patients (2.72\%): 5 had hemoptysis ( 3 had hemoptysis before biopsy, and 2 were cured by medication), and the other 5 had pneumothorax (all recov- 
ered 2 days later without medication).

Ultrasonographic characteristics of the pulmonary lesions are shown in Table 2.

Table 2. Ultrasonographic characteristics of the lesions.

\begin{tabular}{|c|c|c|c|c|c|c|}
\hline & \multicolumn{2}{|c|}{ Shape } & \multicolumn{2}{|c|}{ Echogenicity } & \multicolumn{2}{|c|}{ Shape and echogenicity } \\
\hline & I & $\overline{\mathrm{II}}$ & III & $\overline{I V}$ & V & VI \\
\hline Chronic inflammation & 65 & 8 & 63 & 10 & 60 & 13 \\
\hline Tubercle & 12 & 20 & 13 & 19 & 6 & 26 \\
\hline \multirow[t]{2}{*}{ Malignant lesion } & 31 & 204 & 36 & 199 & 12 & 223 \\
\hline & $\mathrm{P}<0.001$ & & $\mathrm{P}<0.001$ & & $\mathrm{P}<0.001$ & \\
\hline
\end{tabular}

I = cuniform shape; II = other shape; III = with hyperechoic structure scattered in hypoechoic mass; IV = without hyperechoic structure scattered in hypoechoic mass; V = combined I and III; VI = all other combinations but V.

Most of the chronic inflammatory lesions (60/73) demonstrated cuneiform shape with some hyperechoic faculas within the hypoechoic masses (Figures 1 and 2), whereas most of the malignant lesions showed spherical shape and had no hyperechoic dots within them (Figures 3 and 4).
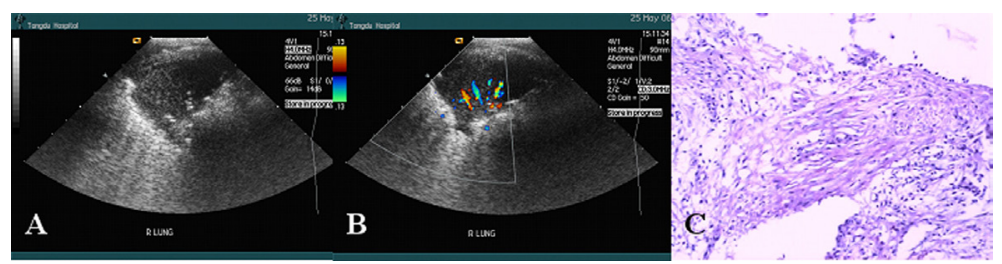

Figure 1. Lesion in the right lung in a 42-year old male patient. A. Gray-scale sonogram of the lesion, showing some hyperechoic structures (extensions of the bronchus) scattered in the hypoechoic and cuniform mass; B. four vessels (Grade III) displayed in the lesions by color Doppler imaging; C. the histopathology indicated chronic inflammation.

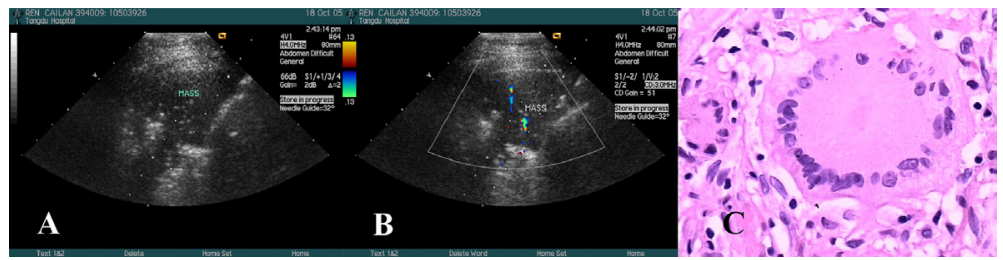

Figure 2. Lesion in the right upper lung in a 61-year old male patient. A. Gray-scale sonogram of the lesion, showing the cuniform shape and some sporadic hyperechoic dots within the lesion (MASS); B. dotted blood flow signals and a long vessel were seen in the lesion by color Doppler imaging; C. the histopathology indicated tubercle.



Figure 3. Lesion in the left lung in a 55-year old male patient. A. Gray-scale sonogram of the lesion, showing a sphere shape and no hyperechoic light spots within the lesion (M); B. there was some short strip vessels in the center of the lesion by color Doppler; C. the histopathology indicated squamous carcinoma. 


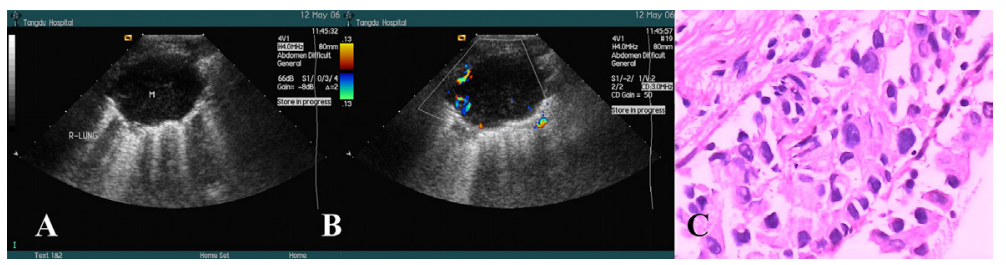

Figure 4. Lesion in the right upper lung in a 63-year old female patient. A. Gray-scale sonogram of the lesion, showing no hyperechoic facula in the globular lesion; B. there was some short strip vessels in the margin of the lesion by color Doppler; C. the histopathology indicated adenocarcinoma.

The validity and reliability of the ultrasound diagnoses of the peripheral pulmonary lesions are shown in Table 3.

Table 3. Validity and reliability of the ultrasound diagnosis methods.

\begin{tabular}{lcrc}
\hline & Shape & Echogenicity & Shape and echogenicity \\
\hline Sensitivity & $66.96(\%)$ & $66.09(\%)$ & $57.39(\%)$ \\
Specificity & $85.02(\%)$ & $82.61(\%)$ & $95.65(\%)$ \\
False-negative rate & $33.04(\%)$ & $33.91(\%)$ & $42.61(\%)$ \\
False-positive rate & $14.98(\%)$ & $17.39(\%)$ & $4.35(\%)$ \\
Positive predictive value & $71.30(\%)$ & $67.86(\%)$ & $88.00(\%)$ \\
Negative predictive value & $82.24(\%)$ & $81.43(\%)$ & $80.16(\%)$ \\
Positive likelihood ratio & 4.47 & 3.80 & 13.20 \\
Negative likelihood ratio & 2.57 & 2.44 & 2.24 \\
Youden's index & 0.52 & 0.49 & 0.53 \\
Consistency rate & $78.57(\%)$ & $76.71(\%)$ & $81.99(\%)$ \\
Kappa value & 0.80 & 0.97 & 0.98 \\
\hline
\end{tabular}

No significant difference was found in blood flow signals between malignant and benign peripheral pulmonary lesions (Table 4).

Table 4. Blood flow signals in the lesions.

\begin{tabular}{lcccc}
\hline & 0 & I & II & III \\
\hline Chronic inflammation & 11 & 26 & 11 & 25 \\
Tubercle & 6 & 14 & 2 & 10 \\
Malignant lesion & 55 & 73 & 26 & 81
\end{tabular}

$\mathrm{P}>0.05$.

\section{DISCUSSION}

In this study, $94.03 \%$ of patients with peripheral pulmonary lesions were correctly diagnosed by ultrasound-guided percutaneous biopsy. Complications occurred in only $2.72 \%$ of the patients, and most of the patients with complications recovered without any medication. These data are similar to the results of other studies (Wang et al., 1995; Middleton et al., 2006; Schuhmann et al., 2014).

On comparison with histological results, individual diagnostic ultrasound characteristics showed low sensitivity but high specificity (Table 2). The highest Youden index value was only 0.53 , whereas kappa values were greater than 0.80 . By observing shape and echo- 
genic characteristics, it is possible to differentiate benign from malignant lesions. A cuneal shape or air bronchogram (Reissig and Kroegel, 2007; Sperandeo et al., 2011; Reissig et al., 2012) within a hypoechoic mass is highly suggestive of chronic inflammation (Figures 1, 2 and Table 3). Anatomically, pulmonary inflammation usually affects a segment or part of a segment of lung tissue, which leads to a cuneiform shape because of the bronchial construction. When lung tissue is inflamed, it develops hyperemia, edema, focal necrosis, or hyperplasia. Some twisted, dilated extensions of the bronchus also occur within the gas of the lesion because of obstruction by the inflammatory substances. On sonograms, these extensions look like scattered "equals" $(=)$ signs. In contrast, a lesion with a non-cuneiform shape and no air bronchogram in it suggests malignancy (Figures 3 and 4). Most malignant lesions present as masses caused by expansive growing and appear globular (Figures 3 and 4). When a mass compresses or obstructs bronchi, the lesion may look cuneiform because of local pulmonary atelectasis; usually, no gas persists in the mass of pulmonary carcinoma. Occasionally, both benign and malignant lesions have cavities that look like irregular, hyperechoic curves in a hypoechoic mass. In the present study, 21 lesions with cavities were found on a CT scan. However, the number was too small to be analyzed.

In early or healing stages of inflammation, many vessels form within the lesion because the function of nervous reflex and vessel activity amines mediator. Most pulmonary carcinomas also develop rich blood supplies, thereby making the color Doppler of little value for distinguishing between benign and malignant pulmonary lesions. Further study is needed regarding the value of pulsed-wave Doppler for such differentiation. However, with color Doppler ultrasound, larger vessels can be displayed, and thus bleeding can be avoided, and proper biopsy specimens can be obtained in most patients.

Several complications occurred in patients in our study. Three patients had preexisting hemoptysis, and their bleeding was aggravated after the ultrasound-guided biopsy. This was likely not due to the biopsy needle invading blood vessels, but rather due to agitation of fragile lesions by the procedure. Two of these patients recovered with medical treatment, and the third was cured without medication. However, hemoptysis in 2 other patients proved to be caused by needle puncture of small vessels. In the 5 patients who developed pneumothorax, all recovered in 2 days without medication. Notably, the pulmonary lesion diameters were $1.9,2.6,1.2,1.2$, and $2.7 \mathrm{~cm}$, respectively; lesions greater than $1.5 \mathrm{~cm}$ in diameter are located only $1.0 \mathrm{~cm}$ away from the pleura. Pneumothorax is often seen in patients with a lesion depth $<1.5 \mathrm{~cm}$, which might be due to the fact that the Tru-Cut needle can easily damage normal lung tissue surrounding lesions at this depth. Associated symptoms are usually very mild. However, hyperechoic gas generated by a pneumothorax can interfere with obtaining an adequate specimen for histopathological analysis.

Despite the complications mentioned above, percutaneous biopsy under the guidance of ultrasound has proved to be a safe, accurate, and cost-effective method for obtaining tissue samples for histopathology diagnosis and could be the first choice for diagnosis of peripheral pulmonary lesions.

\section{ACKNOWLEDGMENTS}

Research supported by the National Natural Science Foundation of China (\#81371566). 


\section{REFERENCES}

Beslic S, Zukic F and Milisic S (2012). Percutaneous transthoracic CT guided biopsies of lung lesions; fine needle aspiration biopsy versus core biopsy. Radiol. Oncol. 46: 19-22.

Bugalho A, Ferreira D, Eberhardt R, Dias SS, et al. (2013). Diagnostic value of endobronchial and endoscopic ultrasoundguided fine needle aspiration for accessible lung cancer lesions after non-diagnostic conventional techniques: a prospective study. BMC Cancer 13: 130.

Chandrasekhar AJ, Reynes CJ and Churchill RJ (1976). Ultrasonically guided percutaneous biopsy of peripheral pulmonary masses. Chest 70: 627-630.

Charboneau JW, Reading CC and Welch TJ (1990). CT and sonographically guided needle biopsy: current techniques and new innovations. Am. J. Roentgenol. 154: 1-10.

Chen A, Hutchings J and Misselhorn D (2010). Ultrasound-guided transthoracic forceps biopsy of a peripheral lung mass. J. Bronchol. Interv. Pulmonol. 17: 84-86.

Feller-Kopman D, Yung RC, Burroughs F and Li QK (2009). Cytology of endobronchial ultrasound-guided transbronchial needle aspiration: a retrospective study with histology correlation. Cancer 117: 482-490.

Haramati LB (1995). CT-guided automated needle biopsy of the chest. Am. J. Roentgenol. 165: 53-55.

Kennedy MP, Jimenez CA, Morice RC and Eapen GA (2008). Ultrasound-guided endobronchial, endoscopic, and transthoracic biopsy. Semin. Respir. Crit. Care Med. 29: 453-464.

Meng XX, Kuai XP, Dong WH, Jia NY, et al. (2013). Comparison of lung lesion biopsies between low-dose CT-guided and conventional CT-guided techniques. Acta Radiol. 54: 909-915.

Middleton WD, Teefey SA and Dahiya N (2006). Ultrasound-guided chest biopsies. Ultrasound Q. 22: 241-252.

Reissig A and Kroegel C (2007). Sonographic diagnosis and follow-up of pneumonia: a prospective study. Respiration 74: 537-547.

Reissig A, Copetti R, Mathis G, Mempel C, et al. (2012). Lung ultrasound in the diagnosis and follow-up of communityacquired pneumonia: a prospective, multicenter, diagnostic accuracy study. Chest 142: 965-972.

Schuhmann M, Bostanci K, Bugalho A, Warth A, et al. (2014). Endobronchial ultrasound-guided cryobiopsies in peripheral pulmonary lesions: a feasibility study. Eur. Respir. J. 43: 233-239.

Seyfarth HJ, Wirtz H, Borte G, Gradistanac T, et al. (2007). Ultrasound guided transthoracic biopsy - safety and efficaces. Pneumologie 61: 563-567.

Shami VM and Waxman I (2005). Technology insight: Current status of endoscopic ultrasonography. Nat. Clin. Pract. Gastroenterol. Hepatol. 2: 38-45.

Sperandeo M, Carnevale V, Muscarella S, Sperandeo G, et al. (2011). Clinical application of transthoracic ultrasonography in inpatients with pneumonia. Eur. J. Clin. Invest. 41: 1-7.

Vilmann P and Puri R (2007). The complete "medical”" mediastinoscopy (EUS-FNA + EBUS-TBNA). Minerva Med. 98: 331-338.

Wang HC, Yu CJ, Chang DB, Yuan A, et al. (1995). Transthoracic needle biopsy of thoracic tumours by a colour Doppler ultrasound puncture guiding device. Thorax 50: 1258-1263. 